\title{
IMPROVING THE PERFORMANCE OF AEROMACS BY COOPERATIVE COMMUNICATIONS
}

\author{
Paola Pulini, German Aerospace Center (DLR), Wessling, Germany \\ Marco Chiani, DEIS/WiLAB, University of Bologna, Cesena, Italy
}

\begin{abstract}
Cooperative communications have gained in the past years an increasing attention, showing promising outcomes on the performance of the systems. The future airport surface communication system represents a potential candidate for the exploitation of these techniques. Focusing on the airport context, we analyze low complexity single-relay cooperative methods. Moreover, we implement a simple amplify and forward scheme showing its performance in the airport communication context.
\end{abstract}

\section{Introduction}

For the near future, a large demand for data exchange within the airport area is expected. The main airport communication target is the guidance and control of the aircrafts. However, also service vehicles (e.g. for luggage handling, fueling, or catering) constitute an important part of the traffic and require a significant capacity. Moreover, additional fixed/portable applications are foreseen, e.g. for connecting remote sensors to the surveillance network of the airport. Nowadays, the only communication infrastructures available at the airports are based on analog systems for voice communications, and the so called VHF digital mode 2 (VDL 2), serving the airport traffic control (ATC) data links. However, the supported data rates are scarce, and the available spectrum in the VHF band is very limited. Therefore, a modern and efficient data link technology in another frequency band is required for the airport area, which allows higher data rates.

During the last World Radio communication Conference (WRC) in 2007, the C-band from $5.091 \mathrm{GHz}$ to $5.150 \mathrm{GHz}$ has been assigned to airport service operations. Based on joint investigations from Eurocontrol and the FAA (Federal Aviation Administration), ICAO (International Civil Aviation Organization) recommended developing a standard for the future Aeronautical Mobile Airport Communications System (AeroMACS) based on the
IEEE 802.16 standard, known as WiMAX [1]. As baseline, the Orthogonal Frequency-Division Multiple-Access (OFDMA) mode of the WiMAX standard is chosen for AeroMACS. OFDMA is wellsuited for the airport environment which is characterized by multipath propagation, moderate Doppler effects and shadowing. In line-of-sight (LOS) conditions, OFDMA performs very well. However, under non-LOS conditions system performance improvements are necessary.

One possibility to improve the system performance is to apply spatial diversity techniques, as multiple-input multiple-output (MIMO) schemes or cooperative communication methods. The advantages of multiple-antenna systems have been widely acknowledged. However, in the aeronautical domain the introduction of multiple antenna systems, especially onboard aircrafts is seen to be critical, limiting the applicability of MIMO. Cooperative communication techniques can represent a valid alternative, since they allow single antenna systems to exploit spatial diversity [2]-[7]. Cooperative communications represent a new paradigm based on the utilization of heterogeneous resources in order to increase the overall performance of the system. A virtual antenna array may be created by the combination of antennas of different users, obtaining spatial diversity. The classic relay system can be seen as an example of cooperative communication, where a user of the system acts as a relay forwarding the received signal. However, cooperative communication methods include more possibilities. There are several fashions for cooperating and the "helping" user may elaborate the received signal in many different ways.

In this paper, we analyze low-complexity cooperative communications protocols in the context of AeroMACS. Detect and forward, amplify and forward and coded cooperation methods are investigated, analyzing their properties in the aeronautical context. We propose a realization of a simple cooperative communication scheme for 
improving the performance of AeroMACS in the reverse link (RL). The proposed scheme, based on the amplify and forward method, increases the reliability of the system by means of a relay which simply forwards the source signal to the destination. In our investigation, the relay can be an aircraft. In this way the introduction of a new relay network is avoided. The resulting system can beneficiate of a spatial diversity of order two, which is equivalent to the result obtained with a multiple-antenna system with two antennas. The receiver can perform a simple selection combining of the two received signals or can adopt a more sophisticated algorithm (e.g. maximal ratio combining), which allows exploiting all the received power. However, in order to perform maximal ratio combining (MRC), all the channel components must be known. Hence, it is necessary to estimate not only the resulting channel coming from the combination of the route source-relay-destination, but also the channel relative to the segment relaydestination. In order to properly estimate all the channels, we insert some pilot tones at the relay before amplifying the signal, allowing the destination to coherently sum all the received signals and maximize the performance of the system. Performance results in term of bit error rate (BER) versus signal to noise ratio (SNR) are also provided.

The paper is organized as follows. Section II provides an overview of the principle of cooperative communications and the description of some basic methods. Section III is dedicated to the airport communication system, focusing on its main characteristics and on the implementation aspects of a cooperative communication approach. Section IV illustrates the amplify and forward scheme in detail, analyzing its implementation in AeroMACS. Simulation results are provided in Section $\mathrm{V}$ and conclusions follow in Section VI.

\section{Overview of Cooperative Communications}

In a cooperative communication system, the transmission between the source and the destination is helped by one (or more) partner, which receives the transmitted signal and retransmits to the destination a copy of the signal transmitted by the source. In this way, a distributed MIMO network is created and a diversity gain is obtained.
In a multi-user system, the users can act as cooperating partners creating a cooperative network. Sharing their resources, they obtain better global performance. Moreover, they keep the costs low, since the installation of an ad-hoc relay network is avoided. This solution offers the advantage of flexibility, large number of potential cooperative partners and operational efficiency. The vehicles operating on the airport surface offer furthermore sufficient power for performing rather complex operations. Again, the cooperation among users offers a wide availability of potential terminals out of wich choosing the most appropriate relay (i.e. the one located in the best position with respect to the source and the destination terminals).

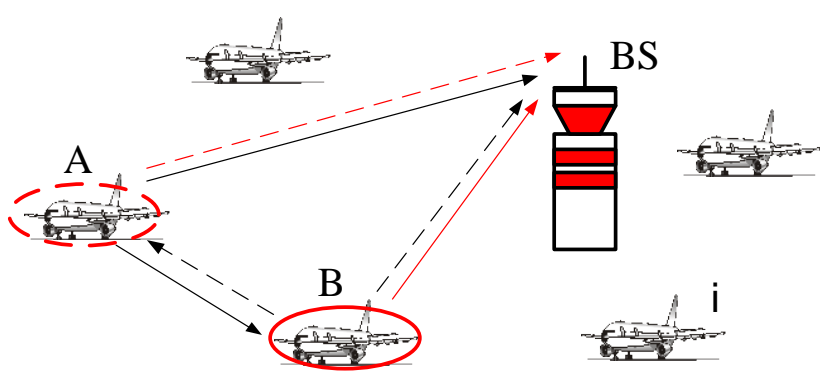

Figure 1: Scheme of cooperation between aircrafts.

Figure 1 represents a scheme of cooperative communication where the users A and B collaborate. In the first case (continuous lines), the user $A$ is helped in the communication with the base station by the user $\mathrm{B}$ (red line). Here A is the source terminal and $\mathrm{B}$ acts as relay transmitting to the base station a replica of the signal transmitted by A. However, the roles can be exchanged and the user A can become the cooperative-terminal that helps $\mathrm{B}$ (dotted lines). In this example the users A and B cooperate with each other. Though, the cooperation can take place not only with cooperating couples of users, the partner assignation can be made in several ways according to different algorithms.

The relay terminal transmits a replica of the signal transmitted by the users according to a certain cooperation method. There are different cooperation techniques characterized by different complexity, modifications required to the system and 
performance. However, all the methods require the receiver separating the original signal from its replicas. The easier way to obtain this separation is to adopt time division, letting source and relay transmitting in different time slots. Table 1 describes an example of single-relay transmission using time division. In the first time slot $\left(\mathrm{t}_{1}\right)$, the source transmits while destination and relay receive. Then, during the second time slot $\left(\mathrm{t}_{2}\right)$, the relay transmits and the destination receives.

Table 1: Cooperation based on time-division.

\begin{tabular}{|l|c|c|}
\hline & $\left(\mathrm{t}_{1}\right)$ & $\left(\mathrm{t}_{2}\right)$ \\
\hline Source & $\mathrm{Tx}$ & Inactive \\
\hline Relay & $\mathrm{Rx}$ & $\mathrm{Tx}$ \\
\hline Destination & $\mathrm{Rx}$ & $\mathrm{Rx}$ \\
\hline
\end{tabular}

\section{Amplify and Forward}

Amplify and forward (AF) methods have been proposed by Laneman in [3] and constitute a simplest form of cooperation between systems. In this case, the relay system only amplifies and retransmits the noisy version of the signal received from the source. The base station receives the different versions of the signal and exploits them for improving the detection of the transmitted bits. Although the noise at the relays is amplified by the cooperation, the system receives independent replicas of the signals increasing the system diversity. For a system with two user cooperating (for example a system with a single relay), a diversity of order two is achieved. Hence, full diversity is attained.

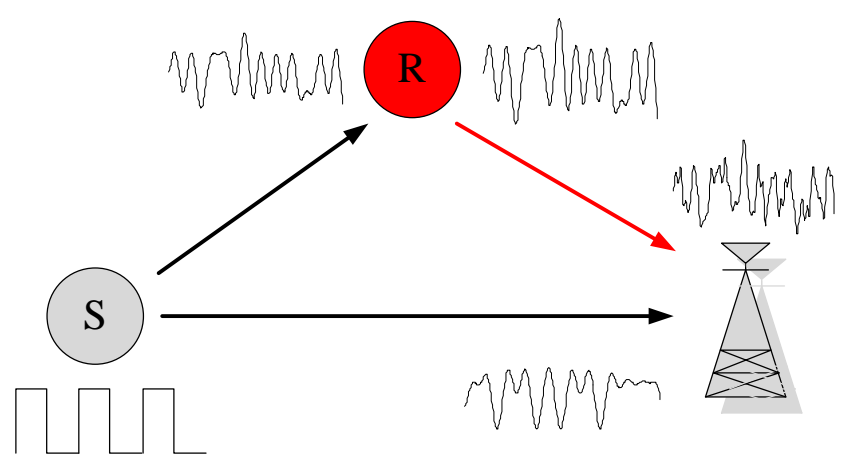

Figure 2: Amplify and forward scheme.

\section{Decode and Forward}

Within the cooperative communication methods, decode and forward (DF) is the one closest to the initial idea of relay system. As represented in Figure 3 , the relay detects, decodes and retransmits the signal transmitted by the source. Sedonaris in [4][5] proposed one of the first example of DF, with decode-and-forward cooperation made by codedivision multiple access (CDMA). In DF, as illustrated in Figure 3, the relay decodes the source's signal and retransmits it after re-encoding the information. The destination receives two independent replicas of the signal that exploits for extracting the information bits. Differently from the previous method, the receiver doesn't require to know the channel between $\mathrm{S}$ and $\mathrm{R}$ to perform a coherent combination of the signals (only the channels between R-S and S-D are required, indeed).

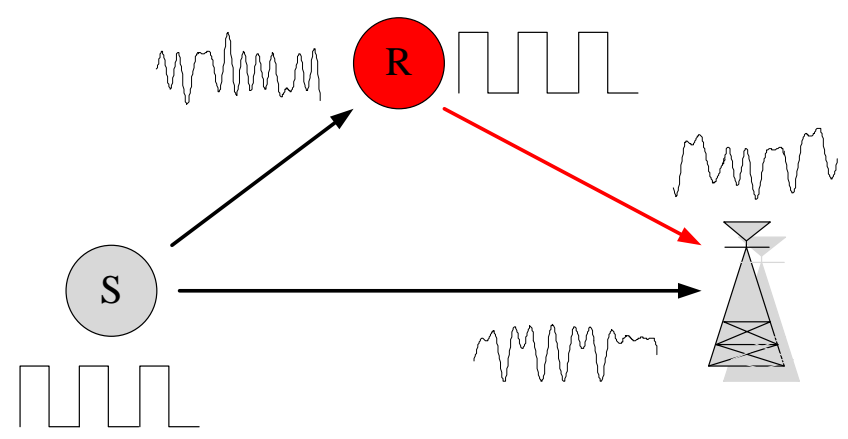

Figure 3: Decode and forward scheme.

\section{Coded Cooperation}

Coded cooperation [8] [9] combines channel coding with cooperation, creating a distributed coding. The basic idea is that each partner transmits a portion of the code, increasing the redundancy. Figure 4 shows a scheme of coding cooperation between two users. Each user encodes their information (the bits of the user A are represented in grey in the figure while the ones of the user $B$ in red) and then divides the codeword into two subsets containing $N_{1}$ and $N_{2}$ bits, respectively. The first subset is transmitted by the original user in the first frame. After decoding the first subset, the collaborating-user can obtain the second subset of the partner and transmits during the second frame. The 
receiver combines the fragments received by the collaborating partners and decodes them jointly obtaining the information bits. Again, including a cyclic redundancy check (CRC) code on the submessages, the partners can independently decide to transmit the second subset of the own message in place of the partner's. In this way, whenever the link between the partners were bad (not allowing a proper decoding and retransmission of the message) the user decides to entirely exploit its resources for itself. Thus, in this case, the system acts as non-cooperative. The partners are hence able to decide whenever it is convenient cooperating or not ${ }^{1}$.

This method represents an attractive cooperation option, thus it achieves gains, while preserving information rate, transmit power, and bandwidth of the non-cooperative system.

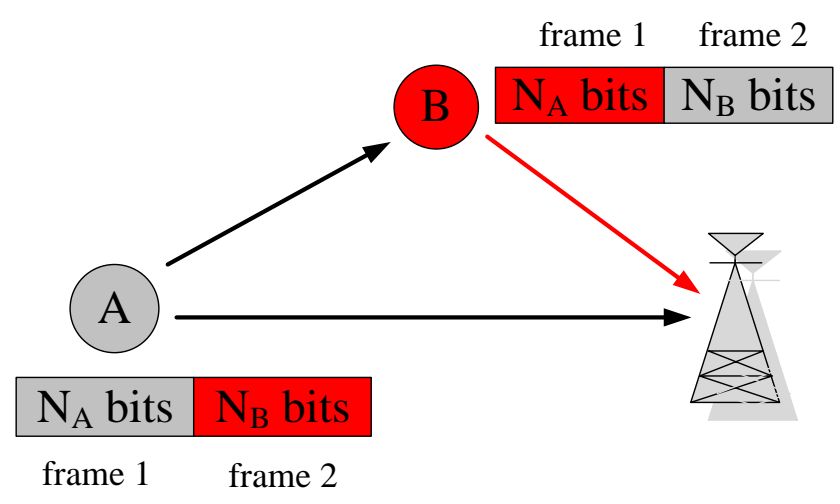

Figure 4: Coded cooperation scheme.

\section{Cooperation in the Airport Environment (AeroMACS)}

In a multi-user system, the users can act as cooperating partners in order to share resources and obtain better information transmissions. The creation of a cooperative network represents a convenient option for a multi-user system. In this case in fact, the installation of an expensive ad hoc relay network is avoided. Besides, the system users offer the great advantage of having already integrated all the devices

${ }^{1}$ This decision is indeed autonomous between partners. The “cooperation mode” doesn't require a centralized decision system. necessary for transmitting and receiving signals. Hence, different types of cooperation algorithms are implicitly allowed. Again, the cooperation among users offers a large range of available terminals within choosing the most appropriate relay.

AeroMACS beneficiates of a large population of aircraft and represents a good candidate for the use of cooperative communications.

Within the airport network, the aircraft could cooperate with each other increasing the performance of the system. The large population of aircraft could offer a wide range of potential relays, out of which choosing in a real time fashion the best one.

At the airport, the ideal application scenario for cooperative communications is represented by the parking scenario. In fact, in this case the aircraft is often in non-line-of-sight with the control tower and the performance of the system tends to suffer for limited time and frequency diversity. Moreover, the network topology (including aircraft and control tower) is static, allowing reliable estimations of the relevant channel gains. Hence, the application of cooperative communications could largely improve the quality of the transmission.

The best cooperating condition is represented by a relay whose link towards the destination presents a better SNR with respect to the one provided by the direct link between source and destination. Such situation may be represented by a relay in line of sight with the user and eventually also with the control tower (and hence with a high SNR in correspondence of both the S-R and R-D links, as depicted in Figure 5).

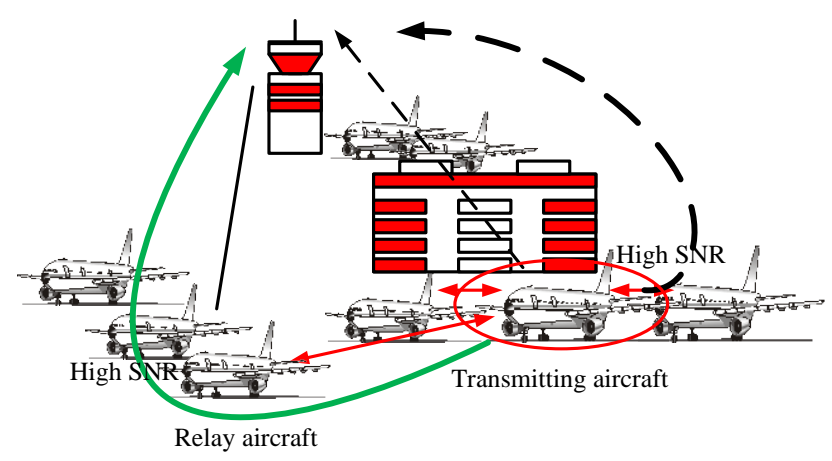

Figure 5: Cooperation between aircraft at the airport. 
In order to select the most appropriate aircraftrelay, the user could (independently from the control tower) exchange messages with the potential relays. Besides, it is possible to exploit the characteristics of the airport topology. Since the airport vehicles are parked according to a specific scheme, this could be used to simply select a good relay. In fact, aircraft parked close to each other guarantee a link between them with high SNR. Hence, it is possible to group potential cooperating-aircraft a priori and then, the active user can independently choose a relay between the group's partners. The selection of the relay can also be done in a completely centralized way. In this case the base station can decide which users cooperate. The centralization of the cooperation increases the network overhead and the complexity.

The cooperative methods presented in the previous section assume that the original and the relay signals are transmitted orthogonally, hence, that the base station is able to separate them. However, except in [3], where the separation of the signals is performed by CDMA, the division of the signals represents a critical aspect. The easiest way to separate the signals is time division (see Table 1), that distributes the source and relay transmissions in different time interval. Another method is frequencydivision. Though, many systems use different bandwidths for receiving and transmitting, making difficult the implementation of cooperation without changing the hardware.

AeroMACS is a system based on OFDMA which will operate in TDD mode. Although the frame is divided in forward link (FL) and reverse link (RL) sub-frames, the ratio between FL and RL is variable, therefore, the relay can receive even during the RL. AeroMACS provides multiple chances of cooperation by means of time division, OFDMA sub-channel and even frequency sub-channel. In the first case the relay could transmit in a different frame but over the same OFDMA sub-channel used by the source message. Moreover, the relay has also the possibility to transmit over a different OFDMA sub-channels.

\section{System Description (AeroMACS) and Simulation Parameters}

We analyzed the future system for the surface airport communications and in particular we focused on the reverse link case. AeroMACS will be based on the OFDMA mode of the IEEE 802.16 (WiMAX) standard [1] and will operate at $5.2 \mathrm{GHz}$ in time division duplex (TDD) mode. The waveform of the system consists of 512 subcarriers over a bandwidth of $5 \mathrm{MHz}$. Two lateral frequency bands are left unused and only the central sub-carriers are effectively used for data transmission and pilot symbols (except the DC sub-carrier, which is nulled). The used subcarriers may be distributed in the frame according to the partially use of sub-carriers (PUSC) mode. Thus, the frame is organized in tiles (see Figure 7). The cyclic prefix (CP) value is $1 / 8$ of the symbol duration. The basic coding scheme includes convolutional codes (CC) and turbo codes with different coding rates. Optional codes are convolutional turbo codes (CTC) and low density parity check (LDPC) codes. The modulation set for the sub-carriers includes quadrature phase shift keying (QPSK), quadrature amplitude modulation (QAM) with 16 and 64 constellation points (16-QAM and 64-QAM).

Table 2: Simulation System Parameters.

\begin{tabular}{|l|c|}
\hline & RL- OFDMA \\
\hline Bandwidth & $5 \mathrm{MHz}$ \\
\hline FFT size & 512 \\
\hline Symbol time $T_{s}$ (w/o CP) & $102.4 \mu \mathrm{s}$ \\
\hline CP & $1 / 8 T_{s}$ \\
\hline Subcarrier spacing & $10.94 \mathrm{kHz}$ \\
\hline Coding, rate & Convolutional, $1 / 2$ \\
\hline Decoding & Soft Viterbi \\
\hline Modulation & QPSK \\
\hline Channel estimation & ID, LIN (freq. domain) \\
\hline
\end{tabular}

For our simulations we used convolutional coding with rate $1 / 2$ and QPSK subcarrier modulation. We considered ideal channel estimation (ID) and linear interpolation based on the pilot tones and tailored for the WiMAX tile structure. Table 2 summarizes the main system parameters used in the simulations.

\section{Channel Description}

For our investigation we used the stochastic airport channel model presented in [10]. It is based on the wide sense stationary uncorrelated scattering 
(WSSUS) model [11], adapted to the peculiarities of the airport environment as multi-path and Doppler effect. Generally, the airports are characterized by different areas referred to as apron, taxi, parking and runway. These areas correspond to different aircraft conditions and present different propagation conditions. The taxi and runway scenarios represent two movement phases and are characterized by LOS conditions. The parking and apron scenarios correspond to areas close to the buildings and are characterized by no or limited mobility. The presence of the buildings makes the control tower in NLOS and stresses the performance of the system. We focused on the parking scenario, which represents the worst case. We assumed Rice factor $K$ values equal to $0 \mathrm{~dB}$. Table 3 provides the main channel parameters used for the simulations.

Table 3: Channel Parameters.

\begin{tabular}{|l|c|}
\hline Scenario & PARKING \\
\hline$K[\mathrm{~dB}]$ & 0 \\
\hline Delay spread $[\mu \mathrm{s}]$ & 1.25 \\
\hline Doppler spread (min, max) $[\mathrm{Hz}]$ & 10,40 \\
\hline Doppler shift max $\left|f_{D}\right|_{\max }[\mathrm{Hz}]$ & 50 \\
\hline Number of taps & 12 \\
\hline
\end{tabular}

\section{Implementation of an AF Scheme for AeroMACS}

In this work we propose to implement a simple amplify and forward scheme to the AeroMACS system. We consider a single relay system, where an aircraft (partner aircraft) acts as a relay receiving and retransmitting the user's signal.

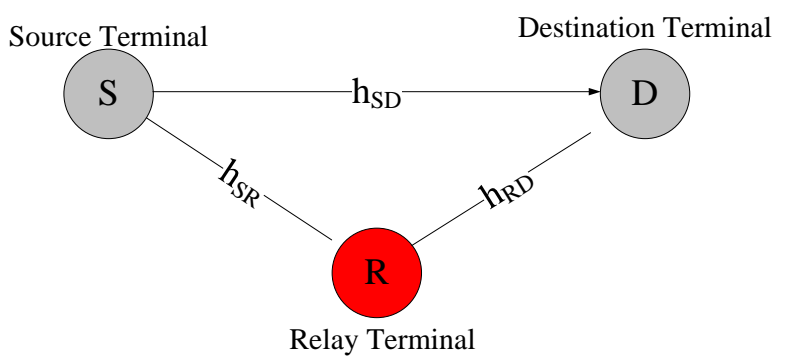

Figure 6: Scheme of a single relay system.
Figure 6 represents a simple amplify and forward scheme, where the communication between a source (S) and a destination (D) is helped by a single relay (R). The relay receives the signal transmitted by the source and retransmits it after an amplification defined by a factor $C$. Assuming the source transmitting only during the first slot, and the relay transmitting on the second slot, the destination receives the signals $y_{S D}$ and $y_{R D}$ during the first and the second slot, respectively. The system may be thus modeled as

$$
\left\{\begin{array}{l}
y_{S D}=h_{S D} x+n_{S D} \\
y_{S R}=h_{S R} x+n_{S R} \\
y_{R D}=h_{R D} x_{R}+n_{R D}
\end{array}\right.
$$

where $x$ represents the transmitted signal at the source, $h_{S D}, h_{S R}$ and $h_{R D}$ are the fading coefficients corresponding to the source-destination, S-R and R-D links, respectively. The signal retransmitted by the relay $x_{R}$ is the received version of $x$ amplified by a factor $C\left(x_{R}=C\left(x h_{S R}+n_{R}\right)\right)$. It is convenient to call $y_{1}=y_{S D}$ and $y_{2}=y_{R D}$ and rewriting equation (1) as:

$$
\left\{\begin{array}{l}
y_{1}=h_{1} x+n_{1} \\
y_{2}=h_{2} x+n_{2}
\end{array}\right.
$$

letting the indexes 1 and 2 represent the direct path S$\mathrm{D}$ and the one provided by the relay S-R-D, respectively. Hence, the signal forwarded by the relay sees a channel $h_{2}=C h_{S R} h_{R D}$, and a noise $n_{2}=C h_{R D} n_{R}+n_{R D}$. All the noise components are modeled as complex Gaussian random variables with zero mean and variances ${\sigma_{S D}}^{2}={\sigma_{1}}^{2},{\sigma_{S R}}^{2}$ and ${\sigma_{R D}}^{2}\left(n_{S D}\right.$ $\sim N\left(0 ; \quad \sigma_{S D}^{2}\right) ; \quad n_{S R} \sim N\left(0 ; \quad \sigma_{S R}{ }^{2}\right) ; \quad n_{R D} \sim N\left(0 ; \quad \sigma_{R D}{ }^{2}\right)$ ). Normalizing the two signals by the relative noise variance $^{2} \sigma_{1}{ }^{2}$ and $\sigma_{2}{ }^{2}$, it is possible to obtain two signals $\dot{y}_{1}$ and $\dot{y}_{2}$ with the same noise variance equal to $1\left(\dot{n}_{1}, \dot{n}_{2} \sim N(0 ; 1)\right)$.

$$
\left\{\begin{array}{l}
\dot{y}_{1}=\frac{y_{1}}{\sigma_{1}}=\dot{h}_{1} x+\dot{n}_{1}=\frac{h_{1}}{\sigma_{1}} \cdot x+\frac{n_{1}}{\sigma_{1}} \\
\dot{y}_{2}=\frac{y_{2}}{\sigma_{2}}=\dot{h}_{2} x+\dot{n}_{2}=\frac{h_{2}}{\sigma_{2}} \cdot x+\frac{n_{2}}{\sigma_{2}}
\end{array}\right.
$$

The system described by the previous equation is equivalent to a generic $1 \mathrm{x} 2$ single input multiple output (SIMO) system, hence, since the optimum

\footnotetext{
${ }^{2}$ Note that if we assume the noise variance for all the systems identical and equal to $\sigma^{2}\left(\sigma_{S D}=\sigma_{R D}=\sigma_{R}=\sigma\right)$, the variances relative to the two paths become $\sigma_{1}{ }^{2}=\sigma_{S D}{ }^{2}=\sigma^{2}$ and $\sigma_{2}{ }^{2}=\sigma^{2}\left(C^{2}\left|h_{R D}\right|^{2}+1\right)$.
} 
weights of the maximum ratio combining (MRC) receiver are given by $w_{1}=\dot{h}_{1} *$ and $w_{2}=\dot{h}_{2} *$ (as for a 1x2 MRC system case). The resulting signal $u$, obtained by the combination of the two received signals $y_{S D}$ and $y_{R D}$, becomes:

$$
u=\dot{h}_{1}^{*} \cdot \dot{y}_{1}+\dot{h}_{2}^{*} \cdot \dot{y}_{2}=\frac{h_{1}^{*}}{\sigma_{1}^{2}} \cdot y_{1}+\frac{h_{2}^{*}}{\sigma_{1}^{2}} \cdot y_{2},
$$

that can be rewritten as

$$
\begin{gathered}
u=\left(\frac{\left|h_{1}\right|^{2}}{\sigma_{1}^{2}}+\frac{\left|h_{2}\right|^{2}}{\sigma_{1}^{2}}\right) \cdot x+\frac{h_{1}^{*} n_{1}}{\sigma_{1}^{2}}+\frac{h_{2}^{*} n_{2}}{\sigma_{1}^{2}}=h_{\text {tot }} \cdot x+n_{\text {tot }} \\
n_{\text {tot }}=\frac{h_{1}^{*} n_{1}}{\sigma_{1}^{2}}+\frac{h_{2}^{*} n_{2}}{\sigma_{1}^{2}}(6) \\
=\frac{\left|h_{1}\right|^{2}}{\sigma_{1}^{2}}+\frac{\left|h_{2}\right|^{2} \sigma_{R D}^{2}}{\sigma_{2}^{4}}+\frac{\left|h_{2}\right|^{2} C^{2}\left|h_{R D}\right|^{2} \sigma_{R}^{2}}{\sigma_{2}^{4}}(7) \\
\sigma_{n_{\text {tot }}}^{2}=\frac{\left|h_{1}\right|^{2}}{\sigma_{1}^{2}}+\frac{\left|h_{2}\right|^{2}}{\sigma_{2}^{2}}(8)
\end{gathered}
$$

Assuming the signal $x$ uniformly distributed with mean power equal to $1 \quad\left(E\left\{|x|^{2}\right\}=1\right)$, the total instantaneous signal-to-noise ratio $\gamma_{\text {tot }}$ becomes:

$$
\begin{gathered}
\gamma_{\text {tot }}=\left(\frac{\left|h_{1}\right|^{2}}{\sigma_{1}^{2}}+\frac{\left|h_{2}\right|^{2}}{\sigma_{2}^{2}}\right)=\gamma_{1}+\gamma_{2}^{3}(9) \\
\gamma_{\text {tot }}=\frac{1}{\sigma^{2}}\left(\left|h_{S D}\right|^{2}+\frac{\left|h_{S R}\right|^{2}\left|h_{R D}\right|^{2}}{\left(\left|h_{R D}\right|^{2}+\frac{1}{C^{2}}\right)}\right)=\gamma_{S D}+\gamma_{S R D} .
\end{gathered}
$$

Hence, at the receiver the resulting instantaneous $\gamma_{\text {tot }}$ is the sum of the two $\gamma s$ relative to the different paths. The first term represents the $\gamma$ relative to the direct link between the source and the destination and corresponds to the performance offered by a single antenna system with no relay. The second term of the equation describes the signal to noise ratio corresponding to the link source-relay-destination and represents the gain, in term of signal-to-noise ratio, introduced by the relay. Focusing on $\gamma_{2}$

$$
\gamma_{2}=\frac{1}{\sigma_{2}}\left(\frac{\left|h_{S R}\right|^{2}\left|h_{R D}\right|^{2}}{\left|h_{R D}\right|^{2}+1 / C^{2}}\right),
$$

\footnotetext{
${ }^{3}$ This result is equal to the one provided by a $1 \times 2$ MRC system.
}

we observe that for high values of $C$ the equation (11) becomes the signal to noise ratio corresponding to the S-R link $\gamma_{S R}$. Hence, the system behaves as a multiple antenna system with two receiving antennas ${ }^{4}$. Vice versa, for $C=0, \gamma_{2}=0$ and the system converts to a non-cooperating system. For $C=1$, as it is reasonable to assume, $\gamma_{2}$ becomes:

$$
\gamma_{2}=\frac{1}{\sigma_{2}}\left(\frac{\left|h_{S R}\right|^{2}\left|h_{R D}\right|^{2}}{\left|h_{R D}\right|^{2}+1}\right) .
$$

From the previous formula it is clear to see that it is important to have the $\gamma$ relative to the link between the source and the relay $\left(\gamma_{S R}\right)$ high. It is trivial to understand that a high $\gamma_{2}$ is fundamental for a good cooperation and to improve the performance of the system. However, as discussed in the previous section, it is possible to adopt some criteria in the relay selection in order to satisfy this request.

\section{On the Channel Estimation}

The previous analysis of the generic amplify and forward system shows that a coherent receiver requires to know all the three different channels (hence, the ones relative to the links S-D, S-R and RD). In order to properly estimate all the channels, the relay could add some pilot tones, according to the scheme illustrated in Figure 7. In this case, the reverse link (RL) of the WiMAX standard is considered. Hence, the frame and the pilot tones positions are based on the tile concept. The frame is divided in tiles, which consist of blocks of 12 subcarriers with pilot sub-carriers at the edges (as illustrated in Figure 7).

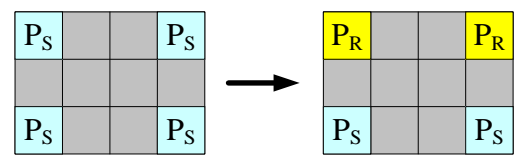

$\mathrm{P}_{\mathrm{S}}$ Pilot subcarrier (source)

Data subcarrier

$\mathrm{P}_{\mathrm{R}}$ Pilot subcarrier (relay)

\section{(a) Tile scheme.}

\footnotetext{
${ }^{4}$ Note that we are neglecting the power used by the relay for retransmitting the source's information. Since $C$ is directly related to the relay power consumption, in a real system, high values of $C$ are unfeasible.
} 


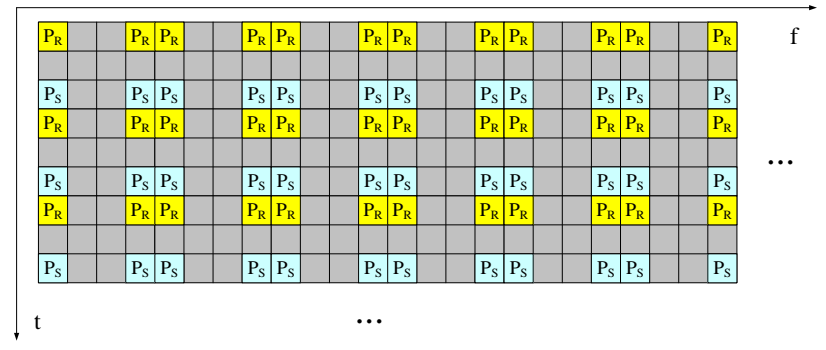

(b) Frame scheme.

Figure 7: Tile (a) and frame (b) schemes of the pilot tones introduction at the relay.

The relay inserts two pilot values in correspondence of the first two pilots of the tile (the ones on the first frequency line) and modify the frame according to scheme presented in the figure. If the channel is sufficiently slow in time, the destination is able to estimate all the channels. In this case, the receiver may perform the channel estimation only in frequency direction, using the two pilots assigned to the link and assuming the channel constant over three consecutive OFDM symbols. The pilots inserted by the relay $(P R)$ to estimate the R-D channel and the pilots of the source (Ps) to estimate $h_{2}$.

\section{Simulation Results}

In this section we present the results obtained by simulations. All the figures show the performance of the system in term of bit error rate (BER) versus average $\mathrm{E}_{\mathrm{b}} \mathrm{N}_{0}$ over the link S-D ${ }^{5}$.

Figure 8 depicts the performance of a generic single-relay amplify and forward system in the AeroMACS context. Here, the channels between the three communicating users are assumed statistically identical and according to the parking scenario [10]. Therefore, the link offered by the relay doesn't provide a better SNR but only a diversity gain. The figure shows the performance with ideal channel estimation and different amplification factors. Three different relay amplification factors are showed for analyzing the system behavior. High values of $C$ are

\footnotetext{
${ }^{5}$ The power used for the relay transmission is not accounted in these figures. However, for a fair comparison it is necessary to account that the total power used for the cooperative transmission is increased with respect to the NON-cooperative system.
}

considered as reference case, although they are unrealistic and do not lead to a fair comparison. ${ }^{6}$. All the cooperative cases (continuous lines) provide a large performance improvement with respect to the non-cooperative case which is represented by the black line $(C=0)$. The cases with high $C$ provide better performance, gaining almost $2 \mathrm{~dB}$ with respect to the case with $C=1$. In fact, as showed in the previous section, increasing the $C$ value, the system becomes equivalent to a single input multiple output (SIMO) system with 2 antennas and MRC receiver. Indeed, the performance of the cooperative system is equal to the one provided by the $2 \times 1$ MRC system. The gains provided by the cooperative schemes are remarkable. Gaining a diversity order equal to 2, they double the slope of the non-cooperative curve (exactly as the multi-antenna case).

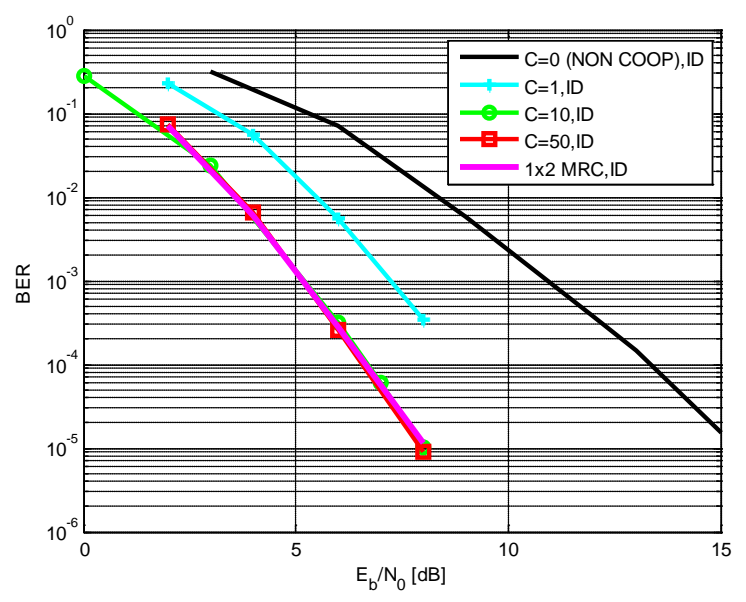

\section{Figure 8: Performance of AeroMACS with AF scheme (ideal channel estimation).}

Figure 9 shows the performance of the system with the proposed way to perform real channel estimation for all the channel coefficients. We used linear channel interpolation based on the pilot tones. The interpolation, done in the frequency domain, is performed in time and frequency direction for the non-cooperative case and only in frequency direction (as described in the previous section) for the cooperative case. The real channel estimation cases (indicated with LIN) are represented by dashed lines,

\footnotetext{
${ }^{6}$ In this work the performance of the system is shown per the link $\mathrm{S}-\mathrm{D}$, indeed. The power used by the relay for helping the user transmission is neglected. However, the total system power shall be controlled and the increase of $\mathrm{C}$ should be avoided.
} 
while the ideal channel knowledge ones (ID) with continuous lines. The introduction of imperfect channel estimation produces a loss of almost $1.6 \mathrm{~dB}$ on the system performance.

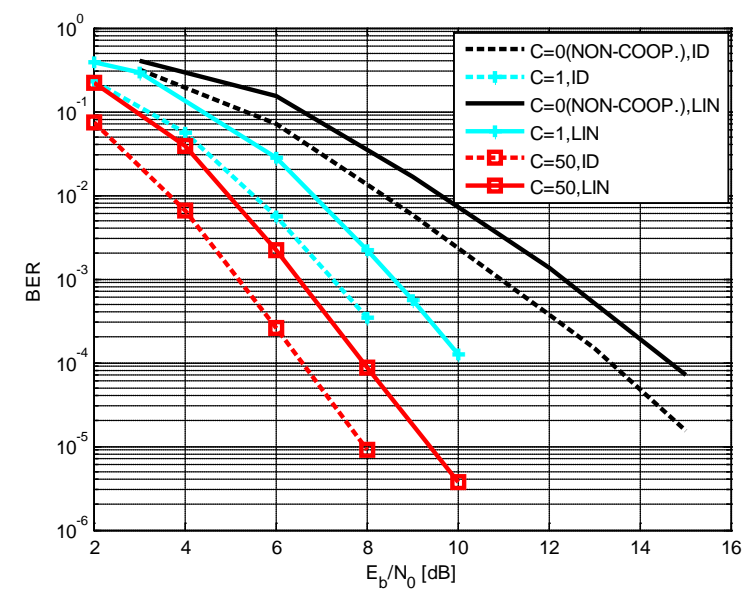

Figure 9: Performance of AeroMACS with AF scheme (realistic channel estimation).

Figure 10 provides the performance of the system with ideal channel knowledge, relay amplification factor $C$ equal to 1 and different cooperation conditions with different channels between source, relay and destination. Besides the case with equivalent channels (light blue curve) considered in the previous figures, two scenarios with different Rice factor $K$ are considered.

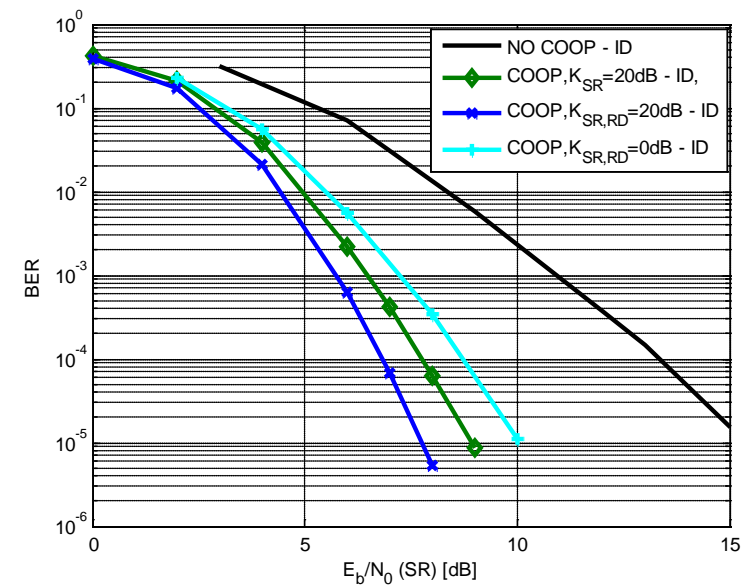

Figure 10: Performance of AeroMACS with AF scheme and different Rice factor $K$ for the relay links (Ideal channel estimation.)
In the first case (green curve), the source and the relay are in line-of-sight $\left(K_{S R}=20 \mathrm{~dB}\right)$. The channel between relay and destination preserves a low Rice factor $K_{R D}=0 \mathrm{~dB}$ (as the one between $\mathrm{S}$ and $\mathrm{D}$ ). Instead, the second case (blue curve) presents high Rice factor in both the links $\left(K_{S R}=K_{R D}=20 \mathrm{~dB}\right)$, as the relay is assumed in line-of-sight with source and destination, both. All the cooperation cases provide large performance gains with respect to the noncooperative case (black curve).

\section{Conclusions}

In this paper, we analyzed the main cooperation methods for the single-rely communications in the context of the future airport surface communication system. We implemented a simple amplify and forward scheme showing its performance. The AeroMACS system can largely beneficiate from the introduction of cooperative communications. The simple amplify and forward scheme presented introduces a diversity gain of order two (the same diversity order obtained by a multiple antenna system with two antennas). Different cooperating methods and configurations could bring even larger benefits to the system performance.

\section{References}

[1] IEEE 802.16-2009, "Standard for Local and Metropolitan Area Networks Part 16: Air Interface.

[2] Cover, Gamal, "Capacity theorems for the relay channel,” IEEE Trans. Inform. Theory, vol. IT-25, pp. 572-584, Sept. 1979.

[3] Laneman, Wornell, Tse, “An Efficient Protocol for Realizing Cooperative Diversity in Wireless network," Proc. IEEE ISIT, Washington, DC, June 2001, p. 294

[4] Sedonaris, Erkip, Aazhang, "User Cooperation Diversity Part I," IEEE Transaction on Comm., vol. 51, no. 11, Nov. 2003, pp. 1927-48.

[5] Sedonaris, Erkip, Aazhang, "User Cooperation Diversity Part II," IEEE Transaction on Comm., vol. 51, no. 11, Nov. 2003, pp. 1927-48.

[6] Laneman, Wornell, "Distributed Space-TimeCoded Protocols for Exploiting Cooperative Diversity in Wireless Networks," IEEE Trans. Info. Theory, vol. 49, no. 10, Oct. 2003, pp. 2415-25. 
[7] Zhao, Valenti, "Some New Adaptive Protocols for the Wireless Relay Channel," Proc. Allerton Conf. Commun., Control, and Comp., Monticello, IL, Oct. 2003.

[8] Hunter, Nosratinia, "Cooperative Diversity through Coding,” Proc. IEEE ISIT, Lousanne, Switzerland, July 2002, p. 220.

[9] Hunter, Nosratinia, "Diversity through Coded Cooperation," IEEE Trans. On Wireless Commun., vol. 5, no. 2, Feb. 2006.

[10] Pulini, "Forward Link Performance Analysis for the Future IEEE 802.16-based Airport Data-Link," in Proc. IEEE Int. Conf. on Communications, Cape Town, South Africa, May 2010.

[11] Bello, "Characterization of randomly timevariant linear channels,” IEEE Trans. Comm., vol. 11, no. 4, pp. 360-393, Dec. 1963.for Fixed Broadband Wireless Access Systems," 2009.

\title{
Email Addresses
}

\author{
Paola.pulini@dlr.de \\ Marco.chiani@unibo.it
}

30th Digital Avionics Systems Conference

October 16-20, 2011 